\title{
Evaluation of Attention and Concentration Using Mobile Computing
}

\author{
Laura-Ivoone Garay-Jiménez ${ }^{\mathrm{a}, *}$, Elena Fabiola Ruiz Ledesma ${ }^{\mathrm{b}}$, Enrique Carmona-García ${ }^{\mathrm{a}}$, \\ Asucena Lozano Gutiérrez ${ }^{c}$, Feggy Ostrosky Shejet ${ }^{\mathrm{c}}$ \\ ${ }^{a}$ Instituto Politécnico Nacional, Unidad Profesdional Interdisciplinaria en Ingeniería y Tecnologías Avanzadas, CDMX, México \\ ${ }^{b}$ Instituto Politécnico Nacional, Escuela Superior de Cómputo, Av. Juan de Dios Bátiz s/n, CDMX, 07320, México \\ ${ }^{c}$ Universidad Nacional Autónoma de México, Facultad de Psicología, CDMX, México \\ "Corresponding author: lgaray@ipn.mx
}

\begin{abstract}
The educational trend toward personalized learning requires the teacher to monitor the learning process continuously. This article presents mobile computing to administer a battery of cognitive tests based on a standard neuropsychological assessment of attention and concentration derived from Neuropsi $\odot$. Currently, specialists perform this test by observing, measuring time, and taking notes of the process to obtain the final scores. Considering the use of this test as an assessment of students' cognitive abilities in a class, the time required for application and evaluation is a challenge itself. As for overcoming this difficulty, the process has been automated through the development of software. The goal is to provide the test to several users simultaneously on their own mobile devices. Then, it is evaluated both attention and concentration on the subject during the solution of the exercises. Variants of the exercises were provided to extent the Neuropsi options. All the collected information is stored on a server. Moreover, the system provides individual and group profiles to the evaluator, such as a teacher or instructor. Likewise, the provided compendium allows the specialist to identify changes in attention and concentration performance and supports their additional recommendations, as well as to go in deep in the research of the cognitive process providing an initial condition evaluation. This work proved that the concept raised by software specialists, designers, and psychologists is feasible into an interdisciplinary team.
\end{abstract}

Keywords - Neuropsychological test; automated evaluation; cognitive skills; education.

\section{INTRODUCTION}

Multiple factors cause the student to have low performance in the learning process, such as misunderstanding of the involved concepts, bad study habits, and insufficient previous knowledge. Hence, this study aims to measure the levels of attention and concentration that the subjects have developed according to their age and educational level [1], [2]. The level of concentration increases just as the student studies are higher because of the complexity of the subjects taught. So, it would be to assume that, to be able to make proper use of the teacher's resources, students need to be conscious of their capability to focus on their studies and self-monitoring their progress.

Nowadays, several commercial software are intended to improve educational performance; cognitive tests and several attention-improving methodologies are also available, such as COGNIFIT [3] or TOWI [4]. Unfortunately, there are neither standards nor clear metrics to allow measuring their performance improvement. Therefore, automatic cognitive tests such as standardized questionnaires are rarely applied to a whole class since more reliable results seem to need a specialist's constant observation forcefully.

On the other hand, there are test applied to groups of different scholarly level or clinical conditions, such as "Psychophysiological Evaluation of Neuropsychological Variables and Cognitive Styles" [4], Casalleto et al.[5] Alternatively, Shubert et al. present further analysis of state of the art [6]. These studies show the cognitive profiles of mental disorders such as depression, anxiety, memory, attention, and concentration problems rather than the subject's cognitive capabilities because their primary objective is to diagnose several diseases. This type of information and that provided by imaging and electroencephalography techniques provide an overview of the function of brain areas and their activity during a specifically requested task.

However, the main limitation for using these studies is the diversity and differences found among individuals and the requirement of a specialized application site. So far, mobile devices have not been used to apply cognitive assessment to 
a group in a classroom setting to establish students' cognitive profiles regarding their attention and concentration skills.

Moreover, even though automated, well-recognized tests for measuring attention and concentration exist. These are addressed to English speakers, such as CANTAB [7], to overcome language differences. Hence, the images are used for cognitive evaluation the assessment scope is reduced to visual performance. Besides, although their translations into Spanish are available, other tests were not explicitly created for Spanish speakers considering the cultural or social traits of Latin America. An overview of several cognitive screening test batteries and neuropsychological test batteries is reviewed by Roebuck et al. [8]. After comparing them, it can be said that the only one that considers Latin American culture is the brief Neuropsi test [9]. However, it is applied in paper format, so a system that would automate the tests contained in this diagnostic battery could be suitable for evaluating a class.

Moreover, based on their research, Ostrosky et al. proposed exercises to improve attention [10]. This system is then focused on evaluating and exercise attention, and this document describes how the system was built, and the results obtained that support the technological viability of this digital implementation.

\section{A. Concepts Associated with Learning}

1) Intelligence: It is defined as the set of capabilities that let the human adapt and solve his life's problems in the practical and theoretical sense through thoughts, besides logical and rational action. One of the definitions of the current concept is based on the research of Stern and Grabner as cited by Shearer et al. [11], who established that it is the ability to adapt conscious thought to new demands, as well as the ability to be adapted to new tasks and living conditions. Intelligence has prerequisites, such as memory, attention, fixation, psychomotor skills, language, fatigue, motivation, and mood; the poorly development of any of them may be due to physical or biochemical alterations of the central nervous system.

2) Learning: it is the ability to acquire knowledge from the external and internal world. Learning allows the subject to acquire knowledge of the world, and memory allows them to codify, store and recover it. The environment modifies the individual's behavior through learning and memory; these mechanisms differ in each subject depending on genetic factors and their previous experiences, which provide the basis for individuality [12], [13]. A significant amount of what we are is a consequence of what we learn and remember. The mental activities that allow us to interpret the world and act in a very particular way are called "cognition"; researchers recognize some differential patterns of this activity and call them cognitive styles. In certain circumstances, our mental process is inefficient, leading to psychopathology [14]. Because learning is defined as individual cognitive ability, attention, concentration, perception, and memory, among other functions, are relevant in the process [11], [12], [15].

3) Memory: it is the ability to record, store and place in our psyche the events we experience theoretically or experientially, and then remember and use this collection of experiences to face new circumstances. It is known that attention is a prerequisite for memory. If the stimuli are disregarded, they cannot be recorded and stored. Therefore, there is a close relationship between attention and memory; experts claim that attention deficits are responsible for $50 \%$ of memory problems[16].

4) Attention: it is regulated by neurological brain centers that must function correctly. However, attending is a biological process and voluntary psychological behavior, which is governed by the internal variables of the individual and external factors [10]. As a psychological process, attention is characterized by transforming its initial reflection nature to an active interaction with the environment. Generally, it is guided and facilitated by a mediator. Then, attention is a conscious search in the perceptual field, unlike the state of alert or wakefulness, which is associated with the level of consciousness and the perceptual field is briefly perceived unless a relevant stimulus appears and then attention is focused. Thus, attention implies being awake, vigilant, and being able to perceive relevant stimuli and discard irrelevant information [15], [ 16], [17].

Attention is not a unitary construct; instead, it comprises several types of attentional processes associated with different neuronal circuits. To succeed in tasks that require high levels of attention, such as alternating attention and divided attention, it is necessary first to train sustained attention and focused attention, since the latter is the most basic type of attention [10],[14].

- Focused attention: It is the ability to respond to specific visual, auditory, or tactile stimuli. The person must attend to a single source of information and ignore all other stimuli. The alert person, but with insufficient attention or inattentiveness, cannot filter the irrelevant stimuli and, therefore, is distracted by external stimuli (sounds, movements, visual stimuli, among others) that occur around them.

- Sustained attention: Refers to the ability to maintain a consistent behavioral response during continuous and repetitive activity. It is the ability to maintain attention and remain alert to stimuli for extended periods. An alteration in this level of attention is observed in people who can focus on only a task and maintain responses for a short period (seconds to minutes) or who fluctuate in the execution of brief periods. It also includes the notion of mental control or performing memory exercises in tasks that involve manipulating information and keeping it in mind. This kind of attention is generally reinforced in the initial period of formal education.

- Selective attention: This level of attention refers to responding to a specific stimulus and inhibiting all responses to distracting stimuli. It requires the monitoring of many information channels to execute a simple task. Irrelevant stimuli easily attract individuals with deficits at this level. These may include visions, sounds or external activities, as well as internal distractions (thoughts, feelings, or concerns).

- Alternate attention: This level of attention refers to the ability to have mental flexibility, which allows individuals to change their focus of attention and move between tasks with different cognitive requirements and control the information that will be addressed 
selectively. The problems at this level are evident in people who have difficulty changing the attention from one task to another once one of them has started. The demands of real-life at this level of attentional control are widespread.

- Divided attention: This level involves the ability to respond simultaneously to the demands of multiple tasks. It requires attending more than one task at the same time, giving two or more behavioral responses while monitoring two or more stimuli. This attentional ability is required for handling simultaneous demands (driving a car while listening to the radio or conducting a conversation). It may reflect either continuous alternating attention or an implicit and automatic process for at least one of the tasks.

5) Concentration: Concentration or sustained attention is considered the extension of focal attention and filter or selectivity. It is measured by how the subject maintains a particular activity with proper functioning both in intensity and amplitude. The task attended must be complicated or changing so that the mind remains fixed on the event. There must be an appropriate state of alertness, and the stimulus should be attractive or motivating. These functions are more complex than the previous one [18].

Attention and concentration are considered as multidimensional cognitive abilities critical for memory, learning, and other cognitive aspects. Attention acts as a gateway for the flow of information that reaches the brain [6]. They are conditioned by motivational, internal, and external factors, such as overexposing to information, affective states, environmental or physiological conditions, and lack of strategies for searching relevant information, among others in typical health conditions.

Conditions such as stress, anxiety, depression, and tension have adverse effects on our lives and affect attentional levels. There is an inverse relationship between these factors and attention: the more stress, anxiety, depression, and tension, the less attention we can have [10]. As Papageorgiou et al. analyzed, emotional disorders in which attention, metacognition, and depressive rumination or anxious worry are relevant factors, so attention training could reduce selffocus and increase attentional and promoted metacognitive control [19].

On the other side, pathological conditions could severely affect daily life, such as attention-deficit/hyperactivity disorder (ADHD), a neurodevelopmental condition characterized by inattention, hyperactivity, or impulsivity. This clinical entity has been considered into DSM-IV test because it is the most common psychiatric disorder in children (30\% to $60 \%$ ), and its symptoms could persist into adulthood. ADHD symptoms affect social, educational, and workplace functioning and significantly impact the subject's life quality[17],[18],[19]. Attention problems are not limited to the different childhood stages; they are also evident during adolescence, youth, and adulthood.

6) Neuropsychological test and attention: Neuropsychology is a branch of clinical psychology that studies the relationship between the brain's daily function and the nervous system's performance. In clinical neuropsychology, finds out function and dysfunction conditions, evaluates, treats, and rehabilitates individuals with suspected or demonstrated neurological or psychological problems, resorting to several assessment methods.

A neuropsychological assessment is done to acquire knowledge on a subject's mental condition and cognitive status. Therefore, a neuropsychological test should meet psychometric criteria of adequate reliability and validity. Furthermore, according to the level of education and age, norms are essential to accurately describe an individual's cognitive abilities and disabilities [20]. Even if several specialized neurophysiological batteries, this study focuses on attention and concentration, limiting only the closest associated tests in state of the art [5].

- Digit Retention[21],[22],[23].

- Corsi cubes[24].

- Tests of Cancellation or Continuous Execution [23],[5].

- Digit Test Symbol [22].

- Test of Symbols and Digits[5].

- Successive Series[22], [23].

- Surveillance and Performance Tests[5].

- Digit Detection[23].

- Continuous Performance Tests[5].

- Paced Auditory Serial Addition Test [25].

- Trace test [5].

In the educational context, difficulties in learning often come along with low academic success rates due to faults in the student's cognitive process. This condition does not necessarily have to be pathological. However, the early detection of inattention would allow establishing adequate intervention strategies to improve cognitive processes and thus improve student performance.

The Neuropsi (C) Atención y Memoria [21], [23] is an instrument that systematically explores the processes of attention, memory, and executive functions and has normative data according to age ( 6 to 85 years) and educational level ( 0 to 24 years) in Spanish-speaking population. These neuropsychological assessment instruments represent an objective tool that allows determining the state of the attentional processes. However, it is impractical for application to large groups since it is in paper format.

On the other hand, there are screening tests for cognitive performance to overview cognitive functions such as attention and concentration. These are a feasible alternative in terms of administration and very useful for diagnosis and subsequent follow-up, but they can yield a high number of falsepositive[9].

The brief neuropsychological battery has been developed for the Mexican population [9] [23] as a screening test, includes orientation (time, person, space), attention and activation, memory, language (oral and written), visual aspects, spatial and visuoperceptual and executive functions. The battery is composed of tests with high neuropsychological validity[9].

The "NEUROPSI: Brief Neuropsychological Evaluation in Spanish" is a screening battery developed for Spanishspeaking subjects, considering age and education level. This battery assesses orientation, attention and concentration, verbal and visuospatial memory, language, reading and writing skills, and motor and conceptual executive functions. A total score classifies individual cognitive performance into typical or mild, moderate, and severe deficits. The battery also 
includes an individual profile of cognitive functions, noting the subject's skills and disabilities in each of the evaluated areas, based on the Neuropsi scores [9], [23]. Therefore, this evaluation system provides both quantitative and qualitative data for the user to be considered.

\section{MATERIALS AND METHOD}

This work was intended to determine the individual's attention and concentration levels in a class, using a digital test battery with the attention and concentration tests and the general data section and the orientation test, in the same way as in [9]. A report with the automatic evaluation that follows the statistical results by age and education level was generated for personal feedback. Then, during the training with suggested exercises, continuous monitoring of the golden metrics was collected. Finally, the training results were added to the group information, and they are presented in a dashboard according to the user role, such as student, teacher, or neurophysiologist.

\section{A. Initial Test}

There are three sections were defined to gather baseline information:

- Neuropsi General Data: This section gathers information about the patient regarding age, sex, level of education, dexterity, and medical and neurological observations. No score is assigned. The information obtained about attention and concentration conditions is used to determine if there are pre-existing causes that may affect the obtained results.

- Orientation: The objective is to determine if the subject is oriented in space, person, and time. The score range is $0-6$. In the case of presenting a score lower than 2 , the results obtained when applying the following tests will not be valid.

- Attention and concentration: This Neuropsi section has three different tests. Firstly, the "Backwards Digits" test where the subject repeats a sequence of digits in reverse order (with a maximum of 6 and a minimum of 2 elements). The maximum length of a correct sequence of digits determines the score ranging from 0 to 6 . The "Visual Detection" test requires the subject to look for all the figures in a test sheet that are equal to the figure shown as a model. The maximum score is 4 . Finally, for the test "20 minus 3", the subject is required to do a successive subtraction by 3 , beginning with 20 . The score range is 0 to 5 .

1) Evaluation: Once the test battery is applied, the scores of the tests are automatically carried out and normalized according to the standardized scale proposed [9], [21], [23]. The levels of attention and concentration are obtained. The results are also provided to the teacher or clinic specialist for further analysis if they are required.

2) Exercises: Based on the exercises proposed [8], [10] as a tool for improving the different levels of attention, the system provides a set of exercises. First, the exercise's logic and the aspects to be considered in the evaluation were identified, then a computational algorithm was proposed for dynamic exercise creation. The random selection of the same exercise model variants allows a higher total number of exercises into the software than the amount proposed [10].

A total of 94 exercises were initially considered to improve the attentional levels shown in Table 1. An analysis of them was carried out to determine if their implementation into a Mobile computing system was feasible or not. Moreover, it was analyzed if it was due to some technological limitation. Finally, a total of 29 exercises were not viable, employing the technology present in a traditional mobile device. Therefore, only 65 of the 94 exercises were implemented in the mobile computing system, with the distribution shown in Table 1.

TABLE I

THE NUMBER OF EXERCISES IMPLEMENTED BY ATTENTIONAL LEVELS

\begin{tabular}{ccc}
\hline Attentional Level & $\begin{array}{c}\text { Number of } \\
\text { exercises }\end{array}$ & $\begin{array}{c}\text { Number of implemented } \\
\text { exercises }\end{array}$ \\
\hline Focused & 1 & 1 \\
Sustained & 24 & 18 \\
Selective & 33 & 29 \\
Alternate & 19 & 13 \\
Divided & 17 & 4 \\
\hline
\end{tabular}

The number of exercises selected to improve attention and concentration on the paper format is limited because it has a static format for each exercise. The specialist generated them with specific figures and number of elements, and they are no longer modified because they are part of a paper book. By generating these exercises on a digital application, it is possible to change the figures, colors, or position, and the final user will perceive them as a new exercise without affecting evaluation or usage. This dynamic generation is a very convenient feature, given that for both the focused and divided attention levels, only four original exercises were viable for adapting into digital format.

In the focused attention exercise, there is just one original exercise, so three new variants were proposed. There are a total of 4 final exercises in the system. Table 2 shows a summary of the exercises to which it was possible to add variants and the quantity of proposed variants.

TABLE II

THE QUANTITY OF VARIANTS OF THE EXERCISES PROPOSED BY ATTENTIONAL LEVELS

\begin{tabular}{lll}
\hline \multicolumn{1}{c}{ Proposed exercise } & $\begin{array}{c}\text { Level of } \\
\text { attention }\end{array}$ & Number of variants \\
\hline Directionality above & Focused & 3 \\
Vowel detection * & & 6 \\
Crawling arrows & & 4 \\
Number detention* & Sustained & 8 \\
Quantities Detection* & 54 \\
Small and large Number's & & 3 \\
detection & Selective & 26 \\
Letter's detection & & 6 \\
Reverse Nomination & & 4 \\
Dial small and large & & 8 \\
numbers & Alternate & \\
Letters sorting & Divided & 3 \\
Semantic categories & &
\end{tabular}

A database is available in the system with the resources used, such as figures and sounds. If the specialist requires the generation of a new exercise, the resources are selected randomly using specific algorithms. The different variants of 
possible exercises are defined mainly by the resources existing in the set, and it will be increasing as the psychologist proposes new resources.

In the exercises in which the resources were shown in such a way that each one fulfilled a specific criterion, algorithms capable of maintaining the criterion were established; This proved to be extremely useful in the levels of attention with few exercises, as it gives a greater variety of exercises without increasing the number of exercise models in the system.

\section{B. Design and implementation of the system}

As to implement the tests and exercises, a system that allows users to interact in a mobile site without dependence on the equipment or operative system was proposed. Under the following structure of the system, modularity and scalability are considered as priority functional requirements.

The system provides a specific response for each user role that performs any requests. The system was created and managed through the web2py framework [26], and its functionality was implemented in Python. All the information used and generated by the system will be stored in a MongoDB database [27].

Four roles with different functionalities were established to maintain the security and integrity of the information (Fig. 1).
1) Administrator: $\mathrm{He}$ is responsible for the management of users and roles in the system and statistics of the system's usage, with access to the medical history and sections of the initial test and being able to modify test sections based on the specialist's request.

2) Evaluator: Specialist responsible for creating and managing the initial test and the different sections related to the exercises. Access to the class' results, as well as any results from a specific student. Access to the raw data of the metrics.

3) Student: Subject to whom is applied the initial test; must solve the different exercises for attention and concentration training. Once he solves the initial test, the system automatically performs an analysis of the results and proposes several exercises. They can visualize their results at any moment and can also request to perform specific exercises.

4) Visitor: a person interested in obtaining general information as an overview of the classes' results as a guess. It needs to be previously authorized by the administrator and does not have access to the students' confidential information.

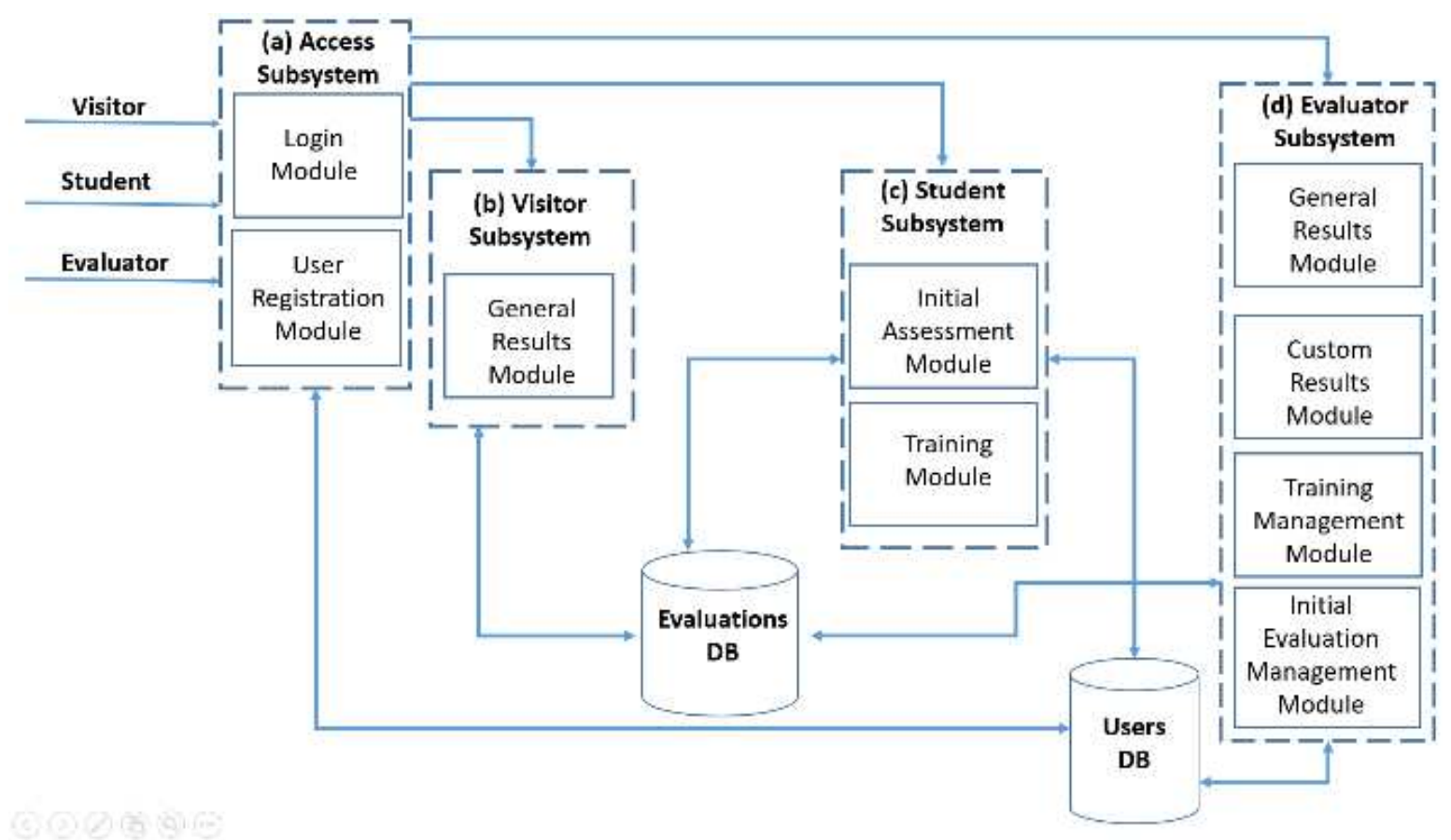

Fig. 1 Scheme of the evaluation and exercise

Each exercise was structured and described according to the attention type intended to improve, the levels of difficulty, and the corresponding evaluation. An example of a specific design is presented in Table 3. For further information about the specific test, the guide for NeuroPsi ${ }^{\circledR}$ should be consulted[9] .

The student has a variety of exercises to do to improve their levels of attention and concentration. An exercise example definition is in Table 3 where each section of the table shows a part of the process and the parameters to be modified. The last sections are related to the automatic evaluation of the participant's performance.
Two aspects must be highlighted: Firstly, if an exercise is requested, the system will create a random variant of the exercise model, which implies that the same user can perform a model exercise with variation each time. In this case, the difference is given by the randomness in which elements of the resources database are extracted. Besides, the placement in the grid is also random.

Secondly, the evaluator consults the information by student or exercise at any difficulty level. The information will be displayed in tables or graphs that are available in a commercial format for further analysis or included in slides of a presentation. 
TABLE III

EXAMPLE OF IMPLEMENTATION OF A PROPOSED EXERCISE WITH THE VARIANT

\begin{tabular}{|c|c|}
\hline \multicolumn{2}{|r|}{ Description of exercise } \\
\hline Name: & Directionality above \\
\hline Attention: & Focused \\
\hline \multicolumn{2}{|r|}{ Description } \\
\hline \multirow{2}{*}{\multicolumn{2}{|c|}{$\begin{array}{l}\text { A grid is shown, and each space has a figure that points to a } \\
\text { specific direction (up, right, down, left). The direction in which } \\
\text { the image points in each space of the grid is determined } \\
\text { randomly and tagged as } 1=\text { up, } 2=\text { right, } 3=\text { down, and } 4=\text { left. } \\
\text { The generated grid must be saved to be able to make the final } \\
\text { evaluation of the exercise. The item must be only one, and it is } \\
\text { selected randomly from the image bank for the exercise. Each } \\
\text { grid space must be a component that allows the user to select by } \\
\text { clicking and determines the spaces that were selected by the user } \\
\text { and the followed sequence, without showing visual changes } \\
\text { when performing this action. }\end{array}$}} \\
\hline & \\
\hline \multicolumn{2}{|c|}{ Description of difficulty levels: } \\
\hline \multicolumn{2}{|c|}{ Initial: $3 \times 3$ grid } \\
\hline \multicolumn{2}{|c|}{ Media: $5 \times 5$ grid } \\
\hline \multicolumn{2}{|c|}{ Advanced: $7 \times 7$ grid } \\
\hline $\begin{array}{l}+2 \text { points fo } \\
-1 \text { point for } \\
-0.5 \text { points }\end{array}$ & $\begin{array}{l}\text { each success } \\
\text { ach error } \\
\text { r each intrusion and omission }\end{array}$ \\
\hline \multicolumn{2}{|c|}{$\begin{array}{l}\text { Evaluation } \\
\text { The record of the user's actions must be saved to make the score } \\
\text { and be able to know the actions that they performed and their } \\
\text { sequence. }\end{array}$} \\
\hline
\end{tabular}

\section{Test and Validation Procedure}

Validation is an essential factor in all software development. It is a process that begins in the early stages of development and continues through the entire life cycle; software testing is the appropriate way to ensure quality in the final product. The software testing procedures applied for this project are described in[28], [29].

Each designed case establishes white-box tests. This test is considered one of the most important types of tests applied to software for obtaining the actual errors in the functionality of the systems' and interactive analysis' performance to eliminate them [28], [29]. In this case, errors are any action that is not considered in the initial design purpose. It also checks that each program instruction is executed at least once.

Performing software tests requires proper planning and sufficient time to complete them. A point to consider is the great variety of existing tests, so it is necessary to determine the appropriate ones for the program's existing needs [29]. Another important aspect is the tools to be used to perform the tests; in this case, the selected tools were Selenium [30] and JMeter [31]. Selenium is a software testing environment for web applications in many programming languages such as Python. Different scripts must be generated using their integrated development environment according to the considered performance test. In the case of the software tests Unit, integration and regression tests were carried out. The Selenium WebDriver was used. The commands are programmed and sent to the browser if the results are returned correctly. On the other hand, the Apache foundation developed JMeter test software, and it is used like Selenium as a loading tool to analyze and measure performance with great emphasis on web applications. The objective is to perform the same tests in the two options to validate the obtained results.

To conclude with the validation test, a small sample of six subjects and two specialists were asked to use the system. The former group was selected into the range of 6-80 years old, considered [9] with studies level over ten years. Table 4 summarizes the conditions of the subject. The latter group oversaw evaluating the results of the modified Neuropsi brief diagnostic battery. These reviewers are part of the original author team with extensive experience applying this test in the paper format.

This last test's objective was to evaluate the proposed system's viability as an auxiliary tool of this neuropsychological non-clinical test. It was confirmed that none of the six subjects selected for the study had a known medical condition that could influence the obtained results. The test was performed in a quiet environment where the subjects were not affected by external factors such as noise or loud sounds that could have altered their attention. They were asked to perform the test in silence or ask in a low voice not to disturb the other participants in the study. The use of devices or mobile applications that were not related to the study was not allowed.

TABLE IV

INFORMATION RELATED TO EACH SUBJECT OF THE STUDY CARRIED OUT

\begin{tabular}{cccccc}
\hline ID & Age & $\begin{array}{c}\text { Studies } \\
\text { (Years) }\end{array}$ & Gender & Culture & Laterally \\
\hline 1 & 12 & 10 & $\mathrm{~F}$ & $\begin{array}{c}\text { Hispanic } \\
\text { (México) }\end{array}$ & $\mathrm{RH}$ \\
2 & 14 & 12 & $\mathrm{~F}$ & $\begin{array}{c}\text { Hispanic } \\
\text { (México) }\end{array}$ & $\mathrm{RH}$ \\
3 & 21 & 17 & $\mathrm{M}$ & $\begin{array}{c}\text { Hispanic } \\
\text { (México) }\end{array}$ & $\mathrm{RH}$ \\
4 & 47 & 20 & $\mathrm{M}$ & $\begin{array}{c}\text { Hispanic } \\
\text { (México) }\end{array}$ & $\mathrm{RH}$ \\
5 & 47 & 25 & $\mathrm{~F}$ & $\begin{array}{c}\text { Hispanic } \\
\text { (México) } \\
\text { Hispanic } \\
\text { (México) }\end{array}$ & $\mathrm{RH}$ \\
6 & 36 & 22 & $\mathrm{MH}$ & $\mathrm{M}$
\end{tabular}

Application of the modified Neuropsi diagnostic battery in its paper version, to all subjects independently. Once the battery was applied for attention and concentration, the subjects were asked to carry out other choice activities for 15 minutes. Meanwhile, two specialists evaluated the diagnostic battery for later comparison with the results obtained by the system.

Subsequently, the six subjects were asked to perform a total of 30 exercises to improve the attention and concentration into the system with unlimited time. If a user makes more exercises than the others, only the first five results registered in the system were considered. They were requested to perform:

- Five exercises of directionality with low difficulty.

- Five exercises of directionality with medium difficulty.

- Five Arrow Tracing exercises with low difficulty.

- Five Arrow Tracing exercises with medium difficulty.

- Five Detection exercises of large and small numbers with low difficulty.

- Five Detection exercises of large and small numbers on medium difficulty 
After the users finished the exercises, they were asked to carry out their choice activities for 15 minutes within the system. Finally, the diagnostic test battery implemented in the mobile system was applied. Because of the size of the sample, a previous Shapiro-Wilk test of normality and likelihood test were performed, an outliners identification with ROUT with $\mathrm{Q}=1 \%$, a method based on the False Discovery Rate (FDR), and a logarithmic transformation were applied if it was required [32]. Then a two one tail t-test looking for statistical equivalence between the test application mode for each test based on Scheuermann method [33] or Two-Way ANOVA for identify the source of the difference and then post-test per subject.

Finally, the global results, age and the period of studies were analyzed, the normality test of the data, the outliners using ROUT WITH $\mathrm{Q}=1 \%$ and a t-student were used to determine if the difference due to this parameter is relevant. Then a Holm-Sidak post-test was done, with alpha=5\%. Each test format was analyzed individually without assuming a consistent SD. Specialists also analyzed the results offered by the system to compare with previous results.

\section{RESULTS AND DISCUSSION}

Once the designed system was tested, errors and non-used code were eliminated, so the system covered all unitary tests as well as the integration and regression tests completely. Both modularity and scalability will allow for the future inclusion of new tests. Because the system offers the advantage of evaluating the tests immediately after the user completes the diagnosis based on the initial battery application, the system provides the results obtained, and it gives suggestions about the exercises to be done based on the results obtained as shown in Figure 2.

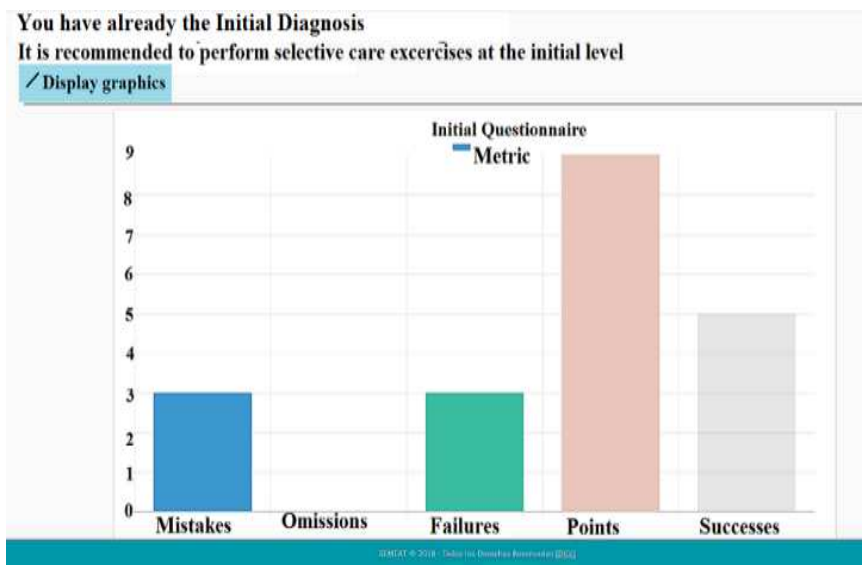

Fig. 2 View of the results of the evaluation and the suggestion of the system to the student

An example of the exercises of directionality available in the system is shown in Figure 3. These exercises are for training the focused attention. Only the different options based on the original exercise are presented [10].

\begin{tabular}{|c|c|c|c|c|}
\hline \multicolumn{5}{|c|}{$\begin{array}{l}\text { SWEMEAT } \\
\leftrightarrow \Rightarrow C \square \text { https: } \| \text { www.semeat.com }\end{array}$} \\
\hline \multicolumn{5}{|l|}{ Excersises } \\
\hline \multicolumn{5}{|c|}{ Selective Sustained Focused } \\
\hline $\begin{array}{c}\text { Right } \\
\text { Directionality }\end{array}$ & Initial & Middle & Advanced & Random \\
\hline $\begin{array}{c}\text { Left } \\
\text { Directionality }\end{array}$ & Initial & Middle & Advanced & Random \\
\hline $\begin{array}{l}\text { Directionality } \\
\text { up }\end{array}$ & Initial & Middle & Advanced & Random \\
\hline $\begin{array}{c}\text { Directionality } \\
\text { below }\end{array}$ & Initial & Middle & Advanced & Random \\
\hline
\end{tabular}

Fig. 3 Focused attention exercise available in the system

The tests' summarized results are presented to the specialist into tables or graphs for an entire class. The guest could observe similar information without the real identity of the subjects. Figure 4 is shown an example of the table of results of a session with four exercises

\begin{tabular}{|c|c|c|c|c|c|c|c|}
\hline \multirow{2}{*}{\multicolumn{8}{|c|}{$\begin{array}{l}\sqrt{\text { SEMEAT }} \\
\Leftrightarrow \Rightarrow C[\text { hatpo:llwww.semeat.com }\end{array}$}} \\
\hline & & & & & & & \\
\hline \multicolumn{8}{|l|}{ Results } \\
\hline Selective & Sostainer & ed Focuse & & & & & \\
\hline \multicolumn{8}{|c|}{ Chip tracing } \\
\hline Date & Level & Correct & Omitted & Mistakes & Error & Points & Time \\
\hline $\begin{array}{l}\text { Jan } 12, \\
2018\end{array}$ & Initial & 18 & 5 & 5 & 10 & 21 & $\begin{array}{c}480.77 \\
\text { seconds }\end{array}$ \\
\hline $\begin{array}{l}\text { Jan } 12, \\
2018\end{array}$ & Initial & 13 & 20 & 1 & 21 & -5.5 & $\begin{array}{l}364.08 \\
\text { seconds }\end{array}$ \\
\hline $\begin{array}{c}\text { Jan } 12, \\
2018\end{array}$ & Initial & o & 18 & 12 & 30 & -45 & $\begin{array}{l}367.44 \\
\text { seconds }\end{array}$ \\
\hline $\begin{array}{l}\text { Jan } 12, \\
2018\end{array}$ & Initial & 13 & 15 & 1 & 16 & 2 & $\begin{array}{c}341.36 \\
\text { seconds }\end{array}$ \\
\hline
\end{tabular}

Fig. 4 Example of a results table of a user performance during a session of sustained attention

In the report of the six subjects' performance for two difficulties levels during the directionality exercises, it is observed that the standard deviation was reduced and all of them improved their punctuation at least one point as shown in table 5, except for subject 4. However, he maintained his performance in two difficulty levels. This information was not previously available, so it requires further analysis by the specialist in a longitudinal protocol.

TABLE V

EXAMPLE OF RESULTS OF THE PERFORMANCE OF THE SIX PARTICIPANTS IN TWO DIFFICULTY LEVELS DURING TRAINING

\begin{tabular}{llllllll}
\hline Subject & \multicolumn{4}{c}{ Low difficulty } & \multicolumn{3}{c}{ Media difficulty } \\
\hline & \multicolumn{1}{c}{$\bar{X}$} & \multicolumn{1}{c}{$s$} & $n$ & $\bar{X}$ & $s$ & $n$ \\
1 & 5.6 & 2.19 & 5 & 8 & 2.44 & 5 \\
2 & 4 & 3.74 & 5 & 10 & 2 & 5 \\
3 & 4.4 & 3.57 & 5 & 7.6 & 2.19 & 5 \\
4 & 6.8 & 2.68 & 5 & 7.6 & 0.89 & 5 \\
5 & 7.2 & 1.78 & 5 & 10.4 & 1.67 & 5 \\
6 & 4.8 & 1.78 & 5 & 9.6 & 2.60 & 5 \\
\hline
\end{tabular}




\section{A. Comparing Digital Application with The Traditional Application}

Table 6 summarizes the paper and pencil test results, and in Table 7, the results when the diagnostic battery was reapplied in digital application mode. A small improvement was expected in the results compared to the previous application. However, the standard deviation was reduced in all subjects along to the several tests and in the global results.

TABLE VI

Results of The Tests IN PENCIL AND PAPER

\begin{tabular}{lllll}
\hline Subject & $\begin{array}{l}\text { Digits } \\
\text { backward }\end{array}$ & $\begin{array}{l}\text { Visual } \\
\text { Detection }\end{array}$ & $\mathbf{2 0 - 3}$ & $\begin{array}{l}\text { Global } \\
\text { Results }\end{array}$ \\
\hline 1 & 4 & 4 & 1 & 9 \\
2 & 4 & 2 & 4 & 10 \\
3 & 6 & 4 & 4 & 14 \\
4 & 5 & 4 & 5 & 14 \\
5 & 3 & 4 & 4 & 11 \\
6 & 4 & 4 & 4 & 12 \\
Average & 4.33 & 3.66 & 3.66 & 11.66 \\
SD & 1.0327 & 0.8164 & 1.3662 & 2.0655 \\
\hline
\end{tabular}

TABLE VII

Tests Results IN THE Digital System

\begin{tabular}{lllll}
\hline Subject & $\begin{array}{l}\text { Digits } \\
\text { backward }\end{array}$ & $\begin{array}{l}\text { Visual } \\
\text { Detection }\end{array}$ & $\mathbf{2 0 - 3}$ & $\begin{array}{l}\text { Global } \\
\text { Results }\end{array}$ \\
\hline 1 & 4 & 4 & 3 & 11 \\
2 & 4 & 4 & 4 & 12 \\
3 & 6 & 4 & 4 & 14 \\
4 & 5 & 4 & 4 & 13 \\
5 & 4 & 4 & 4 & 12 \\
6 & 4 & 4 & 4 & 12 \\
Average & 4.50 & 4.00 & 3.83 & 12.33 \\
SD & 0.83 & 0.00 & 0.40 & 1.03 \\
\hline
\end{tabular}

As was previously reported [9], the effect of a difference in reading and listening abilities on the results of attention is decreased after ten years of studies. Besides, the paper test validation had demonstrated its efficiency in a wide range of age (6 to 85 years old) in screening attention abilities [9], so this sample considered six healthy participants with homogenous abilities of reading (studies period $>10$ years) and experience with computers. The sample age range is 12 to 47 years old into the range of age determined by Neurpsi C. It is essential to highlight that participant attention could have other emotional and previous daily conditions that determine their response, but the exercises' quantity and design are intended to overcome this inconvenience by each participant [9], [10]. The three tests used in this pilot trial are considered into the attention and concentration section of Neuropsic:

1) The original digits backward test: it measures the ability to manipulate verbal information with immediate attention, concentration, and temporary storage. In the digital version, it was transformed into a visual ability task. According to the results previously reported for paper and pencil version, a $5.94 \pm 0.97$ is considered normal for a range of 16-30 years old. Besides a score of $5.58 \pm 0.97$ was reported for 31-74 years old, with over eight years of studies. The results of this study are $4.33 \pm 1.03$ and $4.5 \pm 0.83$ for each application mode. In the first case, the data passed the normality test without outliers. However, the digital application mode has $80 \%$ of its score in 4 , and just two over this value and no outliers were identified. After two-way ANOVA and Sidak test, it was obtained no difference between application mode, but a significant difference among subjects $(\mathrm{p}=0.002)$

2) Visual detection test: the visual selectivity and quickness are evaluated during a visual exploration; an activation-inhibition of quick responses are required to be successful in this type of tasks. This test intends to evaluate the learning process, time of processing information, and sustained attention. These test results are expected to be the maximum scores (4) due to the participants' criterion of inclusion. The results obtained are consistent with previously reported, with no difference among the subjects.

3) The test "20 minus 3": the subject is required to do five successive subtractions. In the original study, this sample condition's performance is considered as usual with 4 to 5 and atypical with less than two. In this case, the results $3.66 \pm 1.36$ and $3.83 \pm 0.40$ for each application mode are equivalents with $95 \%$ of confidence.

The maximum expected was 14-15 points for the global results considering the typical result in Hispanic participants with studies periods over eight years. According to the Shapiro Wilk test and ROUT, these global results have a lognormal distribution with no outliers, so after a logarithmic transformation, and analysis of two-way ANOVA was performed with Sidak multiple comparisons. The mean difference of 0.6667 between the results are not considered significant for application mode, but there was a difference among participants. Besides, $5 / 6$ of the sample increase their performance in the second test.

Finally, using two one side t-test to analyze the two age groups considered previously in [9], both pencil and digital application modes were considered equivalents with $95 \%$ confidence. The modified Neuropsi diagnostic battery results are congruent in the two application modes: Traditional pencil and paper application and using the diagnostic battery included in the developed system.

A significant improvement is introduced when applying the diagnostic battery through the developed system: It allows to measure the total execution times of each test independently and the total time in which the entire test is performed, something that is not accurate enough in the traditional application of pencil and paper, since it is subject to the assessment skills of the specialist who administers it. Besides, metrics sequences are available for the specialist for further analysis.

When applying the diagnostic battery through the developed system, the evaluation criteria [9] are strictly maintained to correct the obtained results, depending on the different application parameters required. This characteristic provides an increase in the accuracy of the evaluation. It immediately offers suggestions for carrying out exercises to improve the levels of attention and concentration included in the system. The latter is not done by the traditional application of the diagnostic battery since it requires additional consultations with the specialist for its realization.

It is essential to point out that these are not a conclusive result for clinical diagnosis since it requires a longer time interval between applications of the test so that the observed improvements are conclusive. This pilot test showed that the 
proposed system could assist a wide range of users, and it could be applied concurrently in 45 minutes to 6 subjects without the presence of the specialist at the site of application.

\section{CONCLUSION}

The proposal of implementation to the exercises based on a primitive model assists the specialist in their creation. Since this system reduces the time used to design and implement the exercises, these can be applied to the student whenever a new version is required. It also showed that it is possible to apply the test in a class simultaneously, and It could reduce the time required to obtain feedback in a teaching process. It will let identify specific cases that may require further attention.

This system will provide new information to the specialist because it gives a more detailed follow-up of the student's performance during the process. In the pilot test, it was expected that a small improvement would be presented by applying the diagnostic battery again after carrying out the improvement exercises. However, not only an improved performance was observed, but the main deviation of the data was also reduced. Then a more accuracy in the responses is obtained.

This work proved that the concept raised by software specialists and designers is feasible into an interdisciplinary team. Therefore, it is a tool for knowing more about the students' cognitive abilities. Future studies must generate more extensive protocols to conclude the application effects of this type of system in the educational field quantify their benefits.

\section{ACKNOWLEDGMENT}

The authors are grateful to the Instituto Politécnico Nacional for supporting this project under the internal financing Institute with contract number: SIP20170314 and SIP2017013.

\section{REFERENCES}

[1] H. Crompton, "Moving toward a mobile learning landscape presenting a mlearning integration framework," Interact. Technol. Smart Educ., 2017.

[2] W. Suryasa, R. Zambrano, J. Mendoza, M. Moya, and M. Rodríguez, "Mobile devices on teaching-learning process for high school level.," Int. J. od Psychosoc. Rehabil., vol. 24, no. 4, pp. 330-340, 2020.

[3] Cognifit, "CogniFit, Test Neuropsicológicos y Estimulación Cognitiva," 2017

[4] P. Studios, "Towi: Desarrollo de habilidades del aprendizaje a través del juego.," SAPI de CV, 2020.

[5] K. B. Casaletto and R. K. Heaton, "Neuropsychological assessment: Past and future," J. Int. Neuropsychol. Soc. JINS, vol. 23, no. 9-10, p. 778, 2017.

[6] A.-L. Schubert and G. T. Frischkorn, "Neurocognitive Psychometrics of Intelligence: How Measurement Advancements Unveiled the Role of Mental Speed in Intelligence Differences," Curr. Dir. Psychol. Sci., vol. 29, no. 2, pp. 140-146, 2020.

[7] C. Cognition, "CANTAB," Cambridge Cognition Ltd.

[8] T. M. Roebuck-Spencer et al., "Cognitive screening tests versus comprehensive neuropsychological test batteries: a national academy of neuropsychology education paper," Arch. Clin. Neuropsychol., vol. 32, no. 4, pp. 491-498, 2017.

[9] M. Ostrosky-Solis, F., A. Ardila A., Roselli, NEUROPSI: Evaluación Neuropsicológica Breve en Español. Mexico: Publingenio, 1998.

[10] F. O. Solís, E. G. Pérez, R. C. Dichy, and J. C. F. Lázaro, ¿Problemas de atención?: un programa para su estimulación y rehabilitación. American Book Store, 2004.

[11] C. B. Shearer and J. M. Karanian, "The neuroscience of intelligence:
Empirical support for the theory of multiple intelligences?," Trends Neurosci. Educ., vol. 6, pp. 211-223, 2017.

[12] D. Schunk, Learning Theories: An Educational Perspective. Pearson, 2019.

[13] B. Byrne, R. K. Olson, and S. Samuelsson, "Behavior-Genetic Studies of Academic Performance in School Students: A Commentary for Professionals in Psychology and Education BT-Reading Development and Difficulties: Bridging the Gap Between Research and Practice," D. A. Kilpatrick, R. M. Joshi, and R. K. Wagner, Eds. Cham: Springer, 2019, pp. 213-232.

[14] M. A. Pievsky and R. E. McGrath, "The neurocognitive profile of attention-deficit/hyperactivity disorder: A review of meta-analyses," Arch. Clin. Neuropsychol., vol. 33, no. 2, pp. 143-157, 2018.

[15] M. Causse, Z. Chua, V. Peysakhovich, N. Del Campo, and N. Matton, "Mental workload and neural efficiency quantified in the prefrontal cortex using fNIRS," Sci. Rep., vol. 7, no. 1, pp. 1-15, 2017.

[16] F. Dolcos et al., "Neural correlates of emotion-attention interactions: From perception, learning, and memory to social cognition, individual differences, and training interventions.," Neurosci. Biobehav. Rev., vol. 108, pp. 559-601.

[17] Y. Luo, D. Weibman, J. M. Halperin, and X. Li, "A review of heterogeneity in attention deficit/hyperactivity disorder (ADHD)," Front. Hum. Neurosci., vol. 13, p. 42, 2019.

[18] D. Stringer et al., "Trajectories of emotional and behavioral problems from childhood to early adult life," Autism, p. 1362361320908972, 2020.

[19] D. R. Coghill, T. Banaschewski, C. Soutullo, M. G. Cottingham, and A. Zuddas, "Systematic review of quality of life and functional outcomes in randomized placebo-controlled studies of medications for attention-deficit/hyperactivity disorder,” Eur. Child Adolesc. Psychiatry, vol. 26, no. 11, pp. 1283-1307, 2017.

[20] P. Julayanont and D. Ruthirago, "The illiterate brain and the neuropsychological assessment: From the past knowledge to the future new instruments," Appl. Neuropsychol. Adult, vol. 25, no. 2, pp. 174 $187,2018$.

[21] D. Ostrosky-Solis, F., Gómez, E., Matute, E., Roselli, M., Ardila, A., Pineda, Neuropsi, Atención y Memoria. Manual e instructivo. Mexico: American Book Store, 2007.

[22] T. Valentine, C. Block, K. Eversole, L. Boxley, and E. Dawson, "Wechsler Adult Intelligence Scale-IV (WAIS-IV)," Wiley Encycl. Personal. Individ. Differ. Meas. Assess., pp. 457-463, 2020.

[23] D. Ostrosky-Solís, F., Gómez, E. Matute, E., M. Roselli, M. Ardila A, Pineda, Neuropsi, Atencion Y Memoria. Manual, Protocolos, Láminas, Tablas Puntuaciones Totales y Perfilesitle. Mexico: American Book Store, 2003.

[24] J. Kamiński, S. Sullivan, J. M. Chung, I. B. Ross, A. N. Mamelak, and U. Rutishauser, "Persistently active neurons in human medial frontal and medial temporal lobe support working memory," Nat. Neurosci., vol. 20, no. 4, pp. 590-601, 2017.

[25] D. L. Woods, J. M. Wyma, T. J. Herron, E. W. Yund, and B. Reed, "The Dyad-Adaptive Paced Auditory Serial Addition Test (DAPASAT): Normative data and the effects of repeated testing, simulated malingering, and traumatic brain injury," PLoS One, vol. 13, no. 4, p. e0178148, 2018.

[26] D. P. M., Web2py, Complete Reference Manual. Web2py, 2020.

[27] S. Bradshaw, E. Brazil, and K. Chodorow, MongoDB: The Definitive Guide: Powerful and Scalable Data Storage. O'Reilly Media, 2019.

[28] G. Fraser and J. M. Rojas, "Software Testing," in Handbook of Software Engineering, Springer, 2019, pp. 123-192.

[29] M. K. Abd Ghani, M. M. Jaber, S. A. Mostafa, A. Mustapha, and M. Abed, "Proper Software Engineering Process in Developing an Integrated Telehealth System," Int. J. Eng. Technol., vol. 7, no. 3.20, pp. 441-450, 2018.

[30] P. Ramya, V. Sindhura, and P. V. Sagar, "Testing using selenium web driver," in 2017 Second International Conference on Electrical, Computer and Communication Technologies (ICECCT), 2017, pp. 17.

[31] S. Matam and J. Jain, Pro Apache JMeter: web application performance testing. Pleasonton, California: Apress, 2017.

[32] D. Lakens, "Equivalence Tests: A Practical Primer for $\mathrm{t}$ Tests, Correlations, and Meta-Analyses," Soc. Psychol. Personal. Sci., vol. 8, no. 4, pp. 355-362, May 2017, doi: 10.1177/1948550617697177.

[33] P. M. Dixon, P. F. Saint-Maurice, Y. Kim, P. Hibbing, Y. Bai, and G. J. Welk, "A Primer on the Use of Equivalence Testing for Evaluating Measurement Agreement.," Med. Sci. Sports Exerc., vol. 50, no. 4, pp. 837-845. 